\title{
The Essential Structures of ISP-I that Influence Serine Palmitoyltransferase Inhibition in Chinese Hamster Ovary Cells
}

\author{
Koji Mizukoshi, ${ }^{*, a}$ Katsuo Matsumoto, ${ }^{a}$ Ryoji Hirose, ${ }^{b}$ and Tetsuro Fujita ${ }^{c}$ \\ ${ }^{a}$ POLA Chemical Industries, Inc.; 560 Kashio-cho, Totsuka-ku, Yokohama 224-0812, Japan: ${ }^{b}$ Mitsui Sugar Co., Ltd.; \\ 2-8-2 Nihonbashi-Honcho, Chuo-ku, Tokyo 103-8423, Japan: and ${ }^{c}$ Research Institute for Production Development; \\ 40-23 Daigokuden Kaide-cho, Muko, Kyoto 617-0004, Japan. Received May 4, 2012; accepted May 22, 2012
}

We investigated the structure-activity relationship between various ISP-I (myriocin, thermozymocidin) analogous which has sphingosine-like structure and serine palmitoyltransferase (SPT) in Chinese hamster ovary (CHO) cells utilizing sphingolipid production as a marker. Our data suggest that the double bond and/ or ketone group within the alkyl chain as well as the alkyl chain are necessary for ISP-I to inhibit SPT. In addition, a serine structure is necessary for SPT inhibitory activity, which confirms previous findings.

Key words ISP-I; myriocin; thermozymocidin; serine palmitoyltransferase; sphingolipid

Serine palmitoyltransferase (SPT) is a rate-limiting enzyme that catalyzes the condensation synthesis of 3-ketodihydroshingosine from palmitoyl $\mathrm{CoA}$ and $\mathrm{L}$-serine. This reaction is a key step in the de novo synthesis of sphingolipids and ceramides. It has been reported that sphingolipids are involved in many cellular events, such as cell proliferation, ${ }^{1,2}$ differentiation $^{3)}$ and cell apoptosis. ${ }^{4,5)}$ Recently, it has been shown that sphingolipids are necessary for cellular infection by the hepatitis $\mathrm{C}$ virus $(\mathrm{HCV}) .{ }^{6}$ Ceramides are a main metabolite of sphingolipids and are known to be an important constituent of intercellular lipids because of their function as the "mortar" in the brick-and-mortar model of the stratum corneum (SC). In addition, ceramides play an essential role in SC barrier function by forming lamellar structures with cholesterol and free fatty acids. ${ }^{7)}$ As mentioned above, sphingolipids and their metabolites play significant roles in a number of organs, which are important for metabolic control.

To date, many compounds have been reported to exhibit SPT inhibitory activity under various experimental conditions.
A competitive inhibition study utilized an SPT protein consisting of the two subunits LCB1 and LCB2 to determine the necessary groups for substrate recognition by SPT. This study by Hanada et al. ${ }^{8)}$ suggested that all of the hydroxyl-, aminoand carboxyl-groups located in L-serine, the first substrate of sphingolipid metabolism, were necessary for SPT substrate recognition. Conversely, a study using intact cells reported ${ }^{9-11)}$ that the sphingosine-like structure was necessary for the inhibition of sphingolipid synthesis. Collectively, these findings suggest that both the serine structure and the sphingosinelike structure are needed for the inhibitory activity of SPT. However, it remains unclear which functional groups of the sphingosine-like structures are necessary for the expression of SPT inhibitory activity.

ISP-I (1) is alternatively referred to as myriocin ${ }^{12,13)}$ or thermozymocidin, ${ }^{14,15}$ ) based on its isolation from Myriococcum albomyces and Mycelia sterilia, respectively. Fujita et al. ${ }^{16)}$ isolated $\mathbf{1}$ as an immunosuppressant from the culture medium of Isaria sinclairii, a fungus found on cicada larva, and has

\begin{tabular}{c|cccc} 
(1: myriocin, \\
thermozymocidin $)$
\end{tabular}

Fig. 1. The Structure of ISP-I (1: Myriocin, Thermozymocidin) and Its Analogous 
been identified as a specific inhibitor of SPT. ${ }^{17}$

In this study, we investigated the relationship between the structure of various $\mathbf{1}$ analogous which has sphingosine-like structure and their SPT inhibitory activity, and we report the findings herein.

\section{MATERIALS AND METHODS}

Compound 1 Analogous All of the compounds examined in this study are shown in Fig. 1. The characteristics of compounds 3-7 have been previously reported ${ }^{18)}$ and compound 2 ((2R)-(E)-2-amino-14-hydroxy-2-hydroxymethy-6-icosenoic acid) was offered from a Institute of Mitsui Sugar Co.

Cell Culture Chinese hamster ovary (CHO)-K1 cells were purchased from Dainippon Pharma Co., Ltd. The cells were maintained in Ham's F-12 medium (Asahi Technoglass, Tokyo, Japan) supplemented with $10 \%$ newborn calf serum (ICN Pharmaceutical, Costa Mesa, CA, U.S.A.), penicillin G and streptomycin sulfate (Invitrogen, NY, U.S.A.). The culture medium was changed to Nutridoma-BO medium (Ham's F-12 medium containing 1\% (v/v) Nutridoma-SP (Boehringer Mannheim, Mannheim, Germany), $10 \mu \mathrm{M}$ of sodium oleate (a complex bovine serum albumin (BSA)) and $10 \mu \mathrm{g} / \mathrm{mL}$ of gentamicin) prior to the addition of the compounds. NutridomaBO medium was used as a sphingolipid-deficient medium. ${ }^{19)}$ All the compounds examined in this study were resuspended in dimethyl sulfoxide (DMSO) (Wako Pure Chemical Industries, Ltd., Osaka, Japan).

Metabolic Labeling of CHO Cell Lipids and Their Separation on TLC Metabolic labeling of $\mathrm{CHO}$ cell lipids with $\mathrm{L}-\left[\mathrm{U}-{ }^{14} \mathrm{C}\right]$ serine (Amersham Pharmacia, Uppsala, Sweden) was carried out as previously described ${ }^{9,20,21)}$ with some modifications. Briefly, $1 \times 10^{6} \mathrm{CHO}$ cells were inoculated and incubated in a $25-\mathrm{mL}$ flask for $24 \mathrm{~h}$. Then, the subconfluent cell monolayer in a $25-\mathrm{mL}$ flask was pre-incubated with $1.5 \mathrm{~mL}$ of Nutridoma-BO medium containing DMSO with or without various compounds at $37^{\circ} \mathrm{C}$ for $1 \mathrm{~h}$. The control vehicle was $0.1 \%(\mathrm{w} / \mathrm{v})$ DMSO. After the addition of $15 \mu \mathrm{L}$ of $\mathrm{L}-\left[\mathrm{U}-{ }^{14} \mathrm{C}\right]-$ serine $(1.85 \mathrm{MBq} / \mathrm{mL})$ (Amersham Pharmacia Biotech), the cells were incubated for $2 \mathrm{~h}$ at $37^{\circ} \mathrm{C}$ for radiolabeling. The incubated $\mathrm{CHO}$ cells were dissolved in $1 \mathrm{~mL}$ of $0.1 \%$ SDS solution, and the lipids were extracted using $3 \mathrm{~mL}$ of chloroform-ethanol $(1: 2)$. The extracted lipids were dried with $\mathrm{N}_{2}$ gas and dissolved in chloroform-ethanol (19:1). Lastly, the samples were spotted and spread on TLC plates with a solvent of methylacetate-1-propanol-chloroform-methanol and $0.25 \%$ $\mathrm{KCl}$ solution $(25: 25: 25: 10: 9, \mathrm{v} / \mathrm{v})$.

Visualization and Image Analysis of the Radiolabeled Lipids Radiolabeled lipids separated on TLC plates were visualized and saved as graphic images with a BAS2000 image analyzer (FUJIFILM Co., Tokyo, Japan). The intensities of the glucosylceramide and sphingomyelin in the graphic images were quantified using Image $\mathrm{J}$ image analysis software. ${ }^{22}$ The relative amount of glucosylceramide and sphingomyelin obtained in each sample was normalized by the DMSO treatment controls.

\section{RESULTS}

The Effect of Compound 1 on de Novo Sphingolipid Biosynthesis in CHO Cells The metabolic labeling of lipids

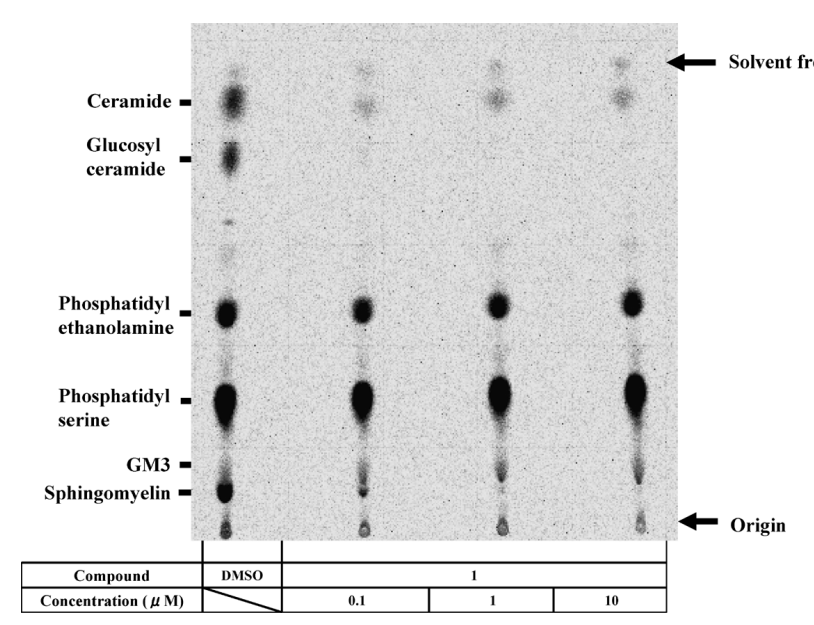

Fig. 2. Effects of Compound $\mathbf{1}$ on de Novo Sphingolipid Synthesis in CHO Cells

$\mathrm{CHO}$ cells were incubated with $\left[{ }^{14} \mathrm{C}\right]$ serine in the presence or absence of $\mathbf{1}$ Lipids were extracted from the CHO cells and separated by TLC. The image above shows the radiolabeled lipids separated on a TLC plate.

with $\left[{ }^{14} \mathrm{C}\right]$ serine revealed that $\mathbf{1}$ inhibited the de novo synthesis of sphingolipids in $\mathrm{CHO}$ cells. Thus, the relative amounts of glucosylceramide and sphingomyelin on the TLC plate decreased in a concentration-dependent manner (Fig. 2). This result is consistent with the previous findings of Hanada et al. ${ }^{9)}$

The Effect of the Compounds 2-7 on de Novo Sphingolipid Biosynthesis in CHO Cells The graphic images of the metabolically radio-labeled sphingolipids visualized on TLC plates are shown in Figs. $3 \mathrm{a}, 4 \mathrm{a}$ and $5 \mathrm{a}$. The relative quantification of glucosylceramide and sphingomyelin spots by image analysis is shown in Figs. 3b, 5b and Figs. 3c-5c, respectively. Compounds 2, 3 and $\mathbf{4}$ showed inhibitory activity against de novo glucosylceramide and sphingomyelin synthesis even at a concentration of $1 \mu \mathrm{M}$ (Figs. 3a-c; Figs. 4a-c). Conversely, compound 5 showed $40 \%$ inhibition of de novo sphingomyelin synthesis and almost $90 \%$ inhibition of glucosylceramide synthesis at a concentration of $100 \mu \mathrm{M}$ (Figs. $4 \mathrm{a}-\mathrm{c}$ ). However, the relative amounts of sphingomyelin and glucosylceramide at $100 \mu \mathrm{M}$ were $60 \%$ and $85 \%$, respectively. This result suggests that 5 possesses almost 100 times lower inhibitory activity than 1. Compound 6 showed inhibition of de novo glucosylceramide and sphingomyelin synthesis at a concentration of $1 \mu \mathrm{M}$ (Figs. 5a-c). This result indicates that $\mathbf{6}$ has the same level of SPT inhibitory activity as $\mathbf{1}$. Alternatively, Compound 7 lacked SPT inhibitory activity (Figs. 5a-c), because the relative intensity of the glucosylceramide and sphingomyelin spots was equal to the control spots.

\section{DISCUSSION}

It has been speculated that both L-serine and a sphingosinelike structure are required for SPT inhibitory activity in cell culture systems. ${ }^{9,11)}$ However, the essential functional groups of the sphingosine-like structure that are necessary for the expression of SPT inhibitory activity have not been elucidated.

Therefore, we sought to elucidate the functional groups of the sphingosine-like structure that are necessary for SPT inhibition in CHO cells. We utilized ISP-I (1) and six compounds, which possess sphingosine-like structures, as SPT inhibitors of glucosylceramide and sphingomyelin synthesis. ${ }^{17)}$ 
a)

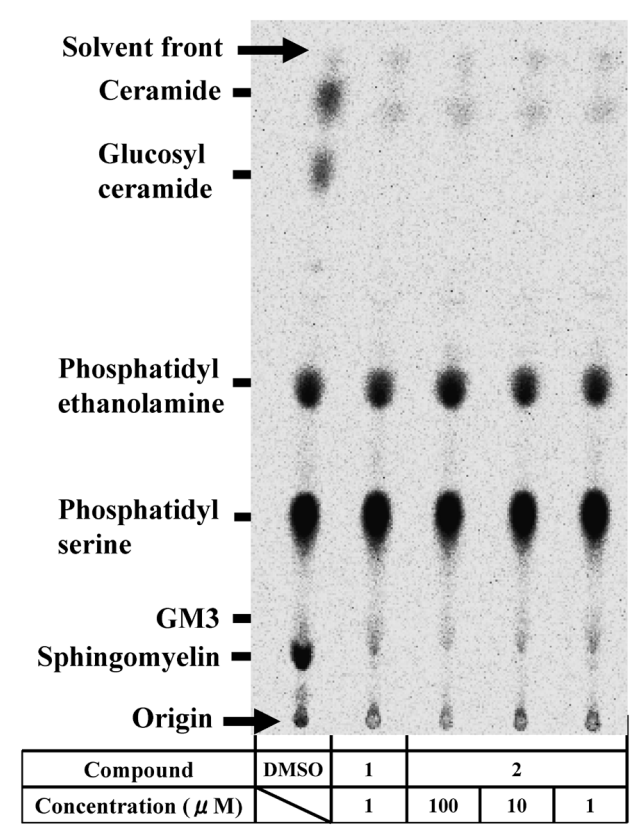

b)

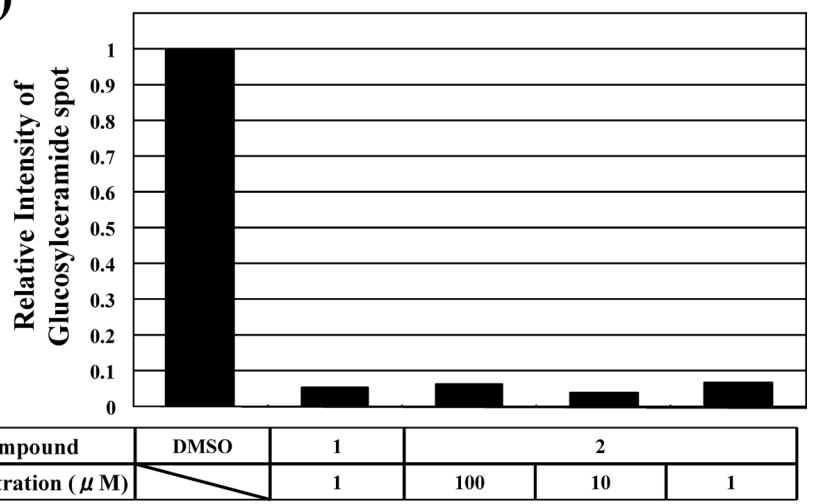

c)

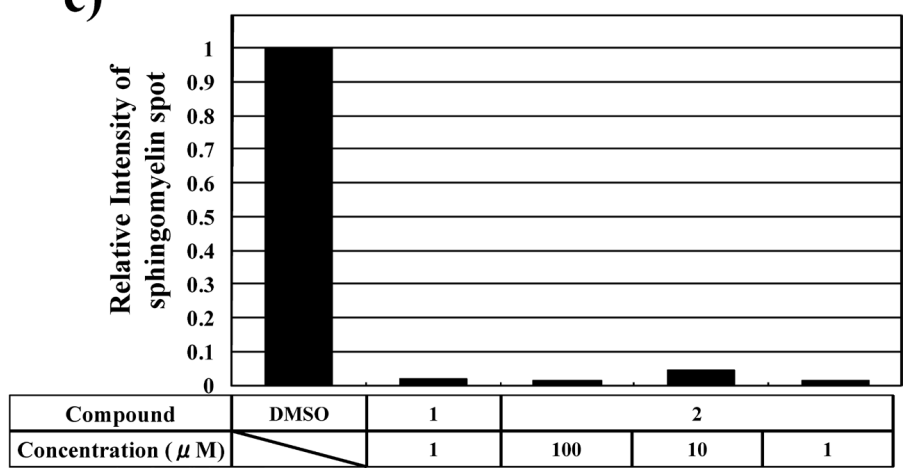

Fig. 3. Effects of Compounds $\mathbf{1}$ and $\mathbf{2}$ on de Novo Sphingolipid Synthesis in CHO Cells

a) $\mathrm{CHO}$ cells were incubated with $\left[{ }^{14} \mathrm{C}\right]$ serine in the presence or absence of $\mathbf{1}$ and $\mathbf{2}$. Lipids were extracted from the cells and separated by TLC. The images of the radiolabeled lipids separated on a TLC plate are shown. b) Spots of glucosylceramide in panel a) were scanned and are represented as the intensity relative to that obtained with DMSO treatment. c) Spots of sphingomyelin in panel A were scanned and are represented as the intensity relative to that obtained with DMSO treatment.

a)

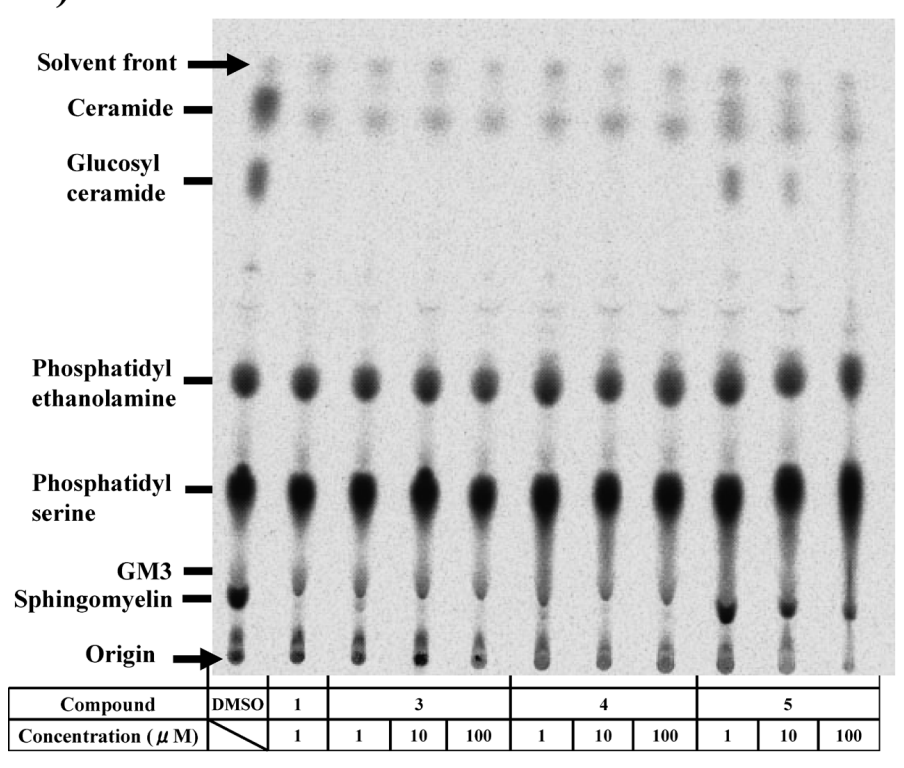

b)

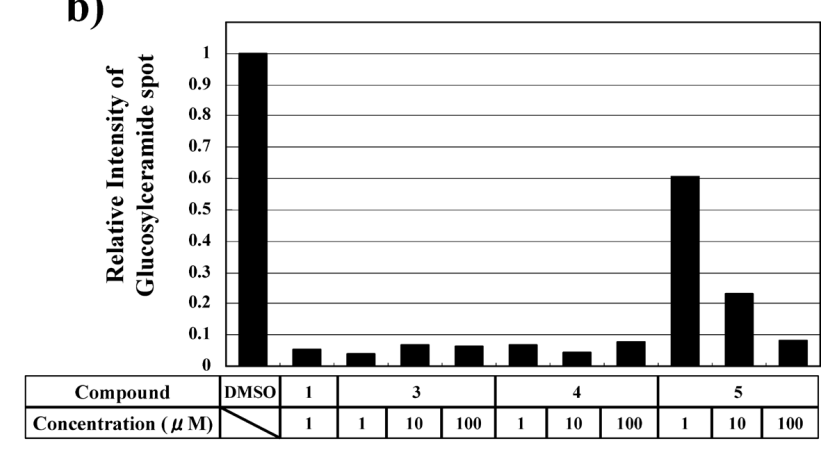

c)

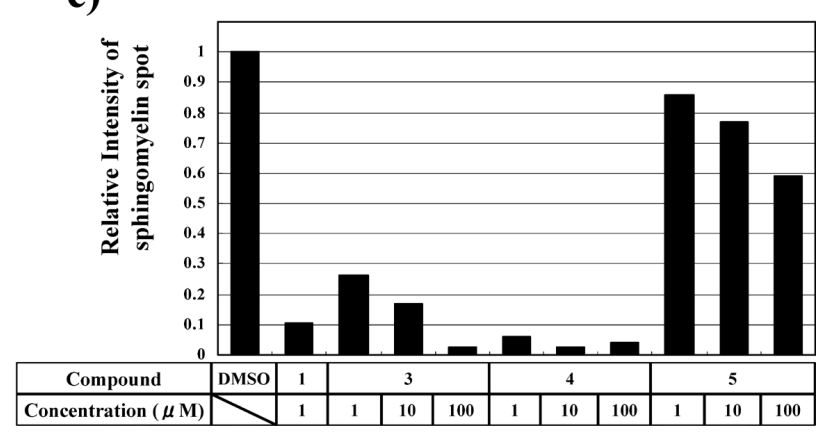

Fig. 4. Effects of Compounds $\mathbf{1}$ and $\mathbf{3}, \mathbf{4}$ and $\mathbf{5}$ on de Novo Sphingolipid Synthesis in CHO Cells

a) $\mathrm{CHO}$ cells were incubated with $\left[{ }^{14} \mathrm{C}\right]$ serine in the presence or absence of ISP-I and its derivatives. Lipids were extracted from the cells and separated by TLC. The images of radiolabeled lipids separated on a TLC plate are shown. b) Spots of glucosylceramide in panel a) were scanned and are represented as the intensity relative to that obtained with DMSO treatment. c) Spots of sphingomyelin in panel A were scanned and are represented as the intensity relative to that obtained with DMSO treatment. 
a)

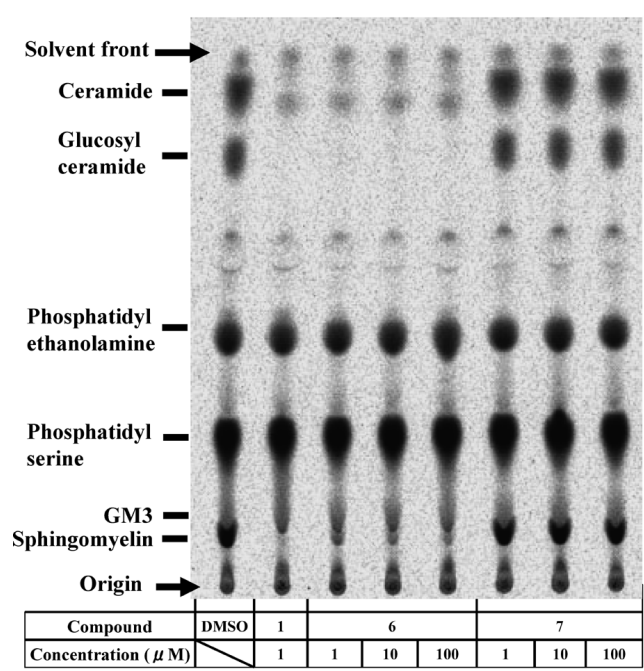

b)

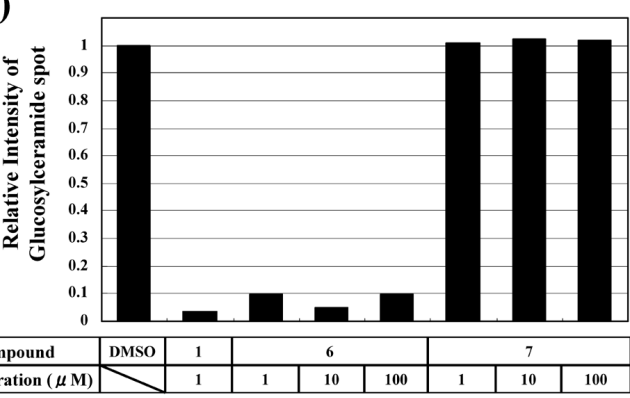

c)

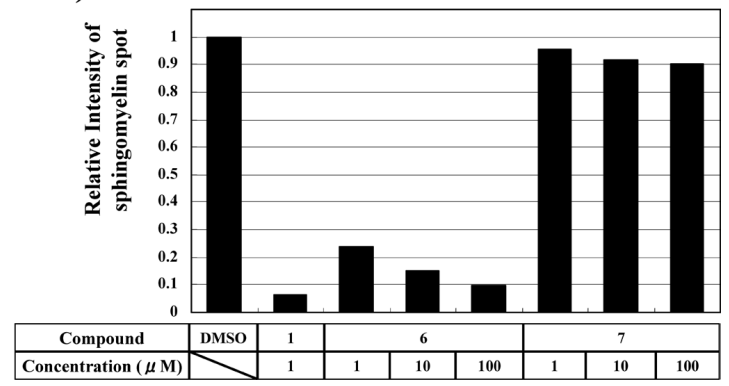

Fig. 5. Effects of Compounds 1 and 6, 7 on de Novo Sphingolipid Synthesis in CHO Cells

a) $\mathrm{CHO}$ cells were incubated with $\left[{ }^{14} \mathrm{C}\right]$ serine in the presence or absence of ISP-I and its derivatives. Lipids were extracted from the cells and separated by TLC. The images of radiolabeled lipids separated on a TLC plate are shown. b) Spots of glucosylceramide in panel a) were scanned and are represented as the intensity relative to that obtained with DMSO treatment. c) Spots of sphingomyelin in panel A were scanned and are represented as the intensity relative to that obtained with DMSO treatment.

The essential functional groups in the sphingosine-like structure of ISP-I that are required for SPT inhibitory activity were as follows: the functional group at the carbon 14 position should be either a ketone or a hydroxyl group as shown with 3, which possessed SPT inhibitory activity at a concentration of $1 \mu \mathrm{M}$ (Figs. $4 \mathrm{a}-\mathrm{c}$ ); the hydroxyl groups at carbons 3 and 4 were not necessary for the expression of SPT inhibitory activity as shown by $\mathbf{2}$, which possessed the same SPT inhibitory activity as 1 (Figs. $3 \mathrm{a}-\mathrm{c}$ ); the double bond at carbon 6 was not necessary as shown by 4 , which displayed SPT inhibitory activity at a concentration of $1 \mu \mathrm{M}$ (Figs. $4 \mathrm{a}-\mathrm{c}$ ); the ketone group at the carbon 14 position was not necessary for SPT inhibitory activity as shown by $\mathbf{6}$, which displayed inhibition of glucosylceramide and sphingomyelin synthesis at a concentration of $1 \mu \mathrm{M}$ (Figs. 5a-c). Conversely, 5 showed $40 \%$ inhibition of de novo sphingomyelin synthesis and $90 \%$ inhibition of glucosyl ceramide synthesis at a concentration of $100 \mu \mathrm{M}$. Therefore, Compound $\mathbf{5}$ possesses an inhibitory activity that is almost 100 times lower than that of $\mathbf{1}$. Therefore, the SPT inhibitory activity of $\mathbf{1}$ is affected by both the double bond at the carbon 6 position and ketone group at the carbon 14 position (Figs. $4 a-c)$.

To determine the relationship between alkyl chain length and SPT inhibitory activity, the effects of 7 were examined, and we found that 7 lacked SPT inhibitory activity (Figs. $5 \mathrm{a}-\mathrm{c})$. This result suggests that alkyl chains longer than the 6th carbon position are necessary for the expression of SPT inhibitory activity, and the alkyl chain length definitely contributes to the expression of SPT inhibitory activity in $\mathbf{1}$.

Table 1 and Fig. 6 show a summary of the functional groups within ISP-I (1) that are necessary to exhibit SPT inhibitory activity. It is known that D-serine competes with L-serine for the amino acid recognition site of isolated SPT, which causes inhibition of sphingolipid production. ${ }^{23)}$ Specifically, the

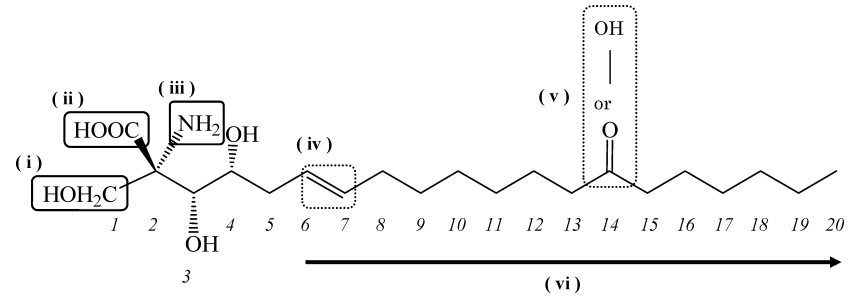

Fig. 6. Essential Functional Groups Needed for the Expression of SPT Inhibitory Activity

Each one of the functional groups marked with a dotted line square, such as (iv) and (v), is necessary. In the straight carbon-chain structure indicated by the solid line, (vi) is necessary. Note: The necessity of the serine residue, including the three functional groups marked with a solid line square ((i)-(iii)), has already been reported.

D-serine structure is necessary for SPT inhibitory activity. However, compound 7 lacked the alkyl chain longer than the 6th carbon position and showed no SPT inhibitory activity in this study (Figs. 5a-c). These results suggest that both alkyl chain length and the serine structure are necessary for the expression of SPT inhibitory activity in CHO cells. Furthermore, it was proposed that both the alkyl chain length and the threedimensional structure of the alkyl chain affect the expression of SPT inhibitory activity as shown by the decrease in the inhibitory activity of $\mathbf{4}$ (Figs. $4 \mathrm{a}-\mathrm{c}$ ).

The differences between the essential functional groups that possess SPT inhibitory activity against the isolated SPT protein in $\mathrm{CHO}$ cells indicates that compounds that have a serine structure can directly interact with the SPT protein and inhibit its activity. ${ }^{23)}$ Alternatively, compounds must pass through the cell membrane and reach the cytoplasm before they can interact with SPT in living CHO cells. It has been reported that carbon chain length affects the cell membrane permeability of compounds. ${ }^{24}$ ) Therefore, the carbon chain 
Table 1. Essential Functional Groups in ISP-I Compounds Needed to Express SPT Inhibitory Activity

\begin{tabular}{cccc}
\hline \hline Derivative & Necessary & Not necessary & Replaceable \\
\hline 2 & & OH at position 3, OH at position 4 & Ketone $\rightarrow$ OH at position 14 \\
3 & Double bond at position 6 & \\
5 & Either ketone at position 14 or & \\
6 & Carbon bond at position 6 & Ketone at position 14 & \\
7 & Cafter the 6th position & & \\
\hline
\end{tabular}

structure longer than the carbon position 6 of 1 seem to contribute to cell membrane permeability and the expression of SPT inhibitory activity.

The findings in this study will contribute to our understanding of the mechanism of SPT compounds and help develop more simple in vivo SPT inhibitors.

\section{REFERENCES}

1) Geilen CC, Barz S, Bektas M. Sphingolipid signaling in epidermal homeostasis. Current knowledge and new therapeutic approaches in dermatology. Skin Pharmacol. Appl. Skin Physiol., 14, 261-271 (2001).

2) Wakita H, Tokura Y, Yagi H, Nishimura K, Furukawa F, Takigawa M. Keratinocyte differentiation is induced by cell-permeant ceramides and its proliferation is promoted by sphingosine. Arch. Dermatol. Res., 286, 350-354 (1994).

3) Spiegel S, Merrill AH Jr. Sphingolipid metabolism and cell growth regulation. FASEB J., 10, 1388-1397 (1996).

4) Ruvolo PP. Ceramide regulates cellular homeostasis via diverse stress signaling pathways. Leukemia, 15, 1153-1160 (2001).

5) Takeda S, Mitsutake S, Tsuji K, Igarashi Y. Apoptosis occurs via the ceramide recycling pathway in human $\mathrm{HaCaT}$ keratinocytes. $J$. Biochem., 139, 255-262 (2006).

6) Umehara T, Sudoh M, Yasui F, Matsuda C, Hayashi Y, Chayama K, Kohara M. Serine palmitoyltransferase inhibitor suppresses HCV replication in a mouse model. Biochem. Biophys. Res. Commun., 346, 67-73 (2006).

7) Elias PM, Feingold KR. Lipids and the epidermal water barrier: metabolism, regulation, and pathophysiology. Semin. Dermatol., 11, 176-182 (1992)

8) Hanada K, Hara T, Nishijima M. Purification of the serine palmitoyltransferase complex responsible for sphingoid base synthesis by using affinity peptide chromatography techniques. J. Biol. Chem., 275, 8409-8415 (2000).

9) Hanada K, Nishijima M, Fujita T, Kobayashi S. Specificity of inhibitors of serine palmitoyltransferase (SPT), a key enzyme in sphingolipid biosynthesis, in intact cells. A novel evaluation system using an SPT-defective mammalian cell mutant. Biochem. Pharmacol., 59, 1211-1216 (2000).

10) Mandala SM, Thornton RA, Frommer BR, Dreikorn S, Kurtz MB. Viridiofungins, novel inhibitors of sphingolipid synthesis. J. Antibiot., 50, 339-343 (1997).

11) Zweerink MM, Edison AM, Wells GB, Pinto W, Lester RL. Characterization of a novel, potent, and specific inhibitor of serine palmitoyltransferase. J. Biol. Chem., 267, 25032-25038 (1992).

12) Kluepfel D, Bagli J, Baker H, Charest MP, Kudelski A. Myriocin, a new antifungal antibiotic from Myriococcum albomyces. J. Antibiot., 25, 109-115 (1972).

13) St-Jacques M. Elucidation of structure and stereochemistry of myriocin. A novel antifungal antibiotic. J. Org. Chem., 38, 12531260 (1973).

14) Craveri R, Manachini PL, Aragozzini F. Thermozymocidin new antifungal antibiotic from a thermophilic eumycete. Experientia, 28, 867-868 (1972).

15) Aragozzini F, Manachini PL, Craveri R, Rindone B, Scolastico C. Isolation and structure determination of a new antifungal $\alpha$-hydroxymethyl- $\alpha$-amino acid. Tetrahedron, 28, 5493-5498 (1972).

16) Fujita $T$, Inoue $K$, Yamamoto $S$, Ikumoto $T$, Sasaki $S$, Toyama $R$, Chiba K, Hoshino Y, Okumoto T. Fungal metabolites. Part 11. A potent immunosuppressive activity found in Isaria sinclairii metabolite. J. Antibiot., 47, 208-215 (1994).

17) Miyake Y, Kozutsumi Y, Nakamura S, Fujita T, Kawasaki T. Serine palmitoyltransferase is the primary target of a sphingosine-like immunosuppressant, ISP-1/myriocin. Biochem. Biophys. Res. Commun., 211, 396-403 (1995).

18) Fujita $T$, Inoue $K$, Yamamoto $S$, Ikumoto $T$, Sasaki $S$, Toyama $R$, Yoneta M, Chiba K, Hoshino Y, Okumoto T. Fungal metabolites. Part 12. Potent immunosuppressant, 14-deoxomyriocin, $(2 S, 3 R, 4 R)$ (E)-2-amino-3,4-dihydroxy-2-hydroxymethyleicos-6-enoic acid and structure-activity relationships of myriocin derivatives. J. Antibiot., 47, 216-224 (1994).

19) Hanada K, Hara T, Fukasawa M, Yamaji A, Umeda M, Nishijima M. Mammalian cell mutants resistant to a sphingomyelin-directed cytolysin. Genetic and biochemical evidence for complex formation of the LCB1 protein with the LCB2 protein for serine palmitoyltransferase. J. Biol. Chem., 273, 33787-33794 (1998).

20) Hanada $K$, Hara $T$, Nishijima $M$, Kuge $O$, Dickson RC, Nagiec MM. A mammalian homolog of the yeast LCB1 encodes a component of serine palmitoyltransferase, the enzyme catalyzing the first step in sphingolipid synthesis. J. Biol. Chem., 272, 32108-32114 (1997).

21) Hanada K, Nishijima M. Selection of mammalian cell mutants in sphingolipid biosynthesis. Methods Enzymol., 312, 304-317 (2000).

22) Abramoff MD, Magelhaes PJ, Ram SJ. Image processing with ImageJ. Biophotonics International, 11, 36-42 (2004).

23) Hanada K, Hara T, Nishijima M. D-Serine inhibits serine palmitoyltransferase, the enzyme catalyzing the initial step of sphingolipid biosynthesis. FEBS Lett., 474, 63-65 (2000).

24) Kamp F, Hamilton JA. How fatty acids of different chain length enter and leave cells by free diffusion. Prostaglandins Leukot. Essent. Fatty Acids, 75, 149-159 (2006). 